\title{
Integrated RF-Photonic Delay Lines using Reconfigurable Photonic Waveguide Meshes
}

\author{
Daniel Pérez, Ivana Gasulla \& José Capmany \\ iTEAM Research Institute \\ Universitat Politècnica de València \\ Valencia, Spain \\ jcapmany@iteam.upv.es
}

\author{
Lee Crudgington, David J. Thomson, Ali Z. Khokhar, \\ $\mathrm{Ke} \mathrm{Li}$, Wei Cao, Goran Z. Mashanovich \\ Optoelectronics Research Centre, \\ University of Southampton, Highfield, Southampton, \\ SO17 1BJ, UK
}

\begin{abstract}
In this paper we demonstrate the implementation of optical delay lines on a programmable hexagonal waveguide mesh integrated in Silicon on Insulator (SOI). This building block enables the synthesis of reconfigurable discrete-time filters and beamforming networks.
\end{abstract}

Keywords-integrated microwave photonics; programmable photonic processors; optical signal processing.

\section{INTRODUCTION}

The tunable delay line is a key basic building block in many applications of Microwave Photonics (MWP) including arbitrary waveform generation, filtering and beamforming [1],[2]. Emerging massive takeover applications, such as 5G communications [3] and the Internet of Things [4], call for a drastic footprint reduction to meet their stringent space, weight and power (SWaP) requirements. Application specific photonic circuits (ASPICs) have been developed capable of implementing MWP delay lines in a variety of particular configurations [5]-[7]. A more versatile alternative is to design a general-purpose integrated programmable photonic processor [8] capable of implementing several delay line architectures by suitable programming of a common photonic hardware architecture. The central element of this processor [9-11] is a reconfigurable optical core built upon a cascade of beamsplitters or Mach Zehnder Interferometers (MZIs) with the incorporation of phase tuning elements that enable independent control of the amplitude and phase of the light [9]. This arrangement can be configured as a cascaded discrete delay line where the light-path can be programmed. The pioneering concept of this waveguide mesh core was proposed in the form of a square lattice by Zhuang and co-workers [9]. More recently, a hexagonal-mesh-based architecture was proposed [10] for the configuration of feed-forward and backward photonic integrated circuits (PICs), featuring improved performance in terms of spatial tuning reconfiguration step, reconfiguration performance, switching elements per unit area and losses per spatial resolution.

In this paper, we report an integrated tunable delay line that is implemented by means of a silicon photonics hexagonal waveguide mesh core. We provide the basic theoretical description and experimental proof of concept, and discuss as well its application to optical beamforming networks.

\section{PRINCIPLE OF OPERATION}

\section{A. Hexagonal waveguide mesh definition}

The proposed processor core is an arrangement of beamspliters with phase shifting capabilities defined as Tunable Basic Units, (TBUs), [10]. The optical interconnection nodes that connect three TBUs, one-by-two, results in a hexagonal waveguide-mesh topology as illustrated in Fig. 1 $(\mathrm{a}, \mathrm{b})$. In the same way as in [9], by biasing the TBUs with the proper electrical signals, the TBU can perform 2 operation modes: as a tunable coupler or as a discrete delay line in cross or bar state, as plotted in Fig. 1(c). The optical signal can be routed over the hexagonal lattice enabling the synthesis of programmed discrete optical delay lines. The combination of tunable couplers and discrete delay lines enables a wide variety of optical processing functionalities like optical filtering, dispersion compensation, true-time delaying and beamforming networks. In the case of tunable and reconfigurable optical filters, the optical response can be directly translated to reconfigurable software-defined RF-photonics filters by employing a modulation/detection scheme with the signal to be filtered.

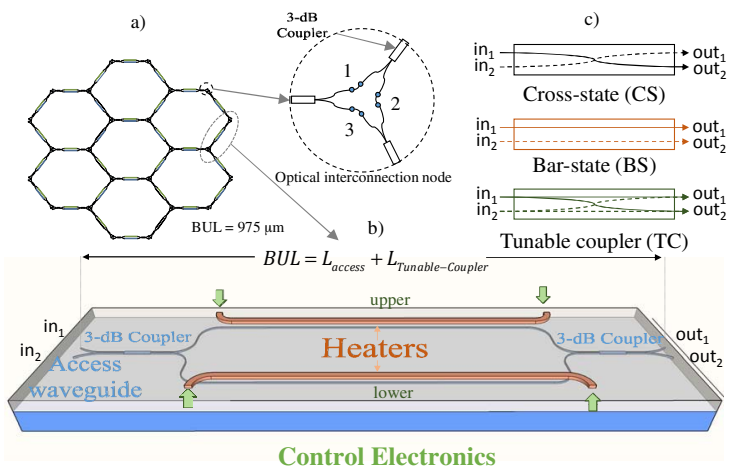

Fig. 1. (a) 7-cell layout of the hexagonal waveguide-mesh core with a zoomed vision of an optical interconnection node and (b) a Tunable Basic Unit (TBU). (c) Three operation modes of the TBU. BUL: Basic Unit Length. 
The TBU illustrated in Fig 1 (b) consists of a $2 \times 2$ balanced MZI loaded with a phase shifter (PS) on each arm. Each thermo-optic PS is implemented by means of a metal heater subject to the Joule effect. Overall, the TBU can synthetize independently the amplitude splitting ratio and the phase response. Next, we demonstrate several selected configuration examples of true-time-delay lines and their application in RF photonic filtering and optical beamforming from the total of over 100 PICs that can be programmed on a 7-cell hexagonal mesh.

\section{FABRICATED DEVICE}

The device of Fig. 2 was fabricated in SOI wafers with a 220 -nm thick silicon overlay and a $3-\mu \mathrm{m}$ thick buried oxide layer. E-beam lithography was employed to write the grating couplers. Dry etching of $70 \mathrm{~nm}$ into the silicon overlay to form the grating couplers was then carried out followed by resist stripping. Another e-beam lithography and 120-nm silicon dry etching step was performed to produce the optical waveguides. Following resist stripping, $1 \mu \mathrm{m}$ of PECVD silicon dioxide was deposited to act as the upper cladding layer of the waveguides. Photolithography was then performed to define isolation trench openings, followed by a deep dry etching process to etch through the top cladding, silicon overlay and buried oxide layer providing thermal isolation to adjacent devices and improved the efficiency of the heaters. A $1.8-\mu \mathrm{m}$ thick metal layer was deposited after the resist had been stripped. A subsequent photolithography and dry etching step realized electrodes used to provide localized heating to tune the devices. The resist was then stripped and the wafers diced into individual dies. These dies were then mounted onto 3 PCBs and a wire bonding process was used to provide electrical connections both within the die and between the die and the PCB.

\section{OPTICAL DELAY LINES}

On-chip optical delay lines can be used to temporally store optical signals and are a key basic building block for optical processing in digital and microwave photonics applications.

We report here the measurements related to the inherent discrete delay line capability of our fabricated hexagonal waveguide processor core. For these results, we have employed an Optical Vector Analyzer (OVA) from LUNA ${ }^{\circledR}$ and a maximum of 18 current sources for the TBU electrical biasing. Discrete delay lines can be programmed by suitably tuning the TBUs to increase the light-path length where the optical signal goes through. The basic time delay corresponding to the fabricated TBUs is $13.5 \mathrm{ps}$, given by a BUL of $975 \mu \mathrm{m}$. As an example, Fig. 3 illustrates two different settings for the 7-cell layout. In the first case, the light is travelling through 5 BULs,
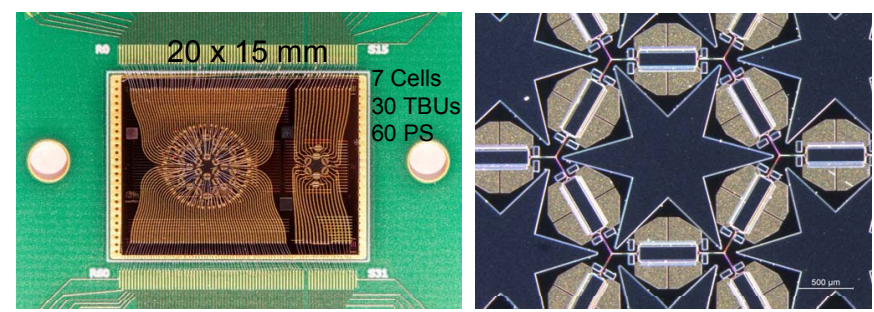

Fig. 2. Fabricated PCB-mounted photonic die (left) and fabricated hexagonal cell image (right).

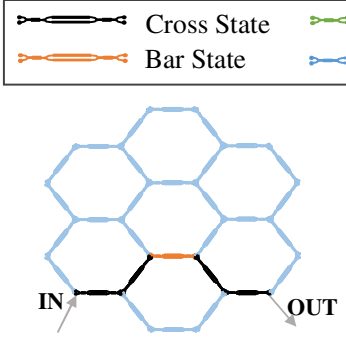

67.5 ps (5 BULs)
Tunable Coupler Not used/Available

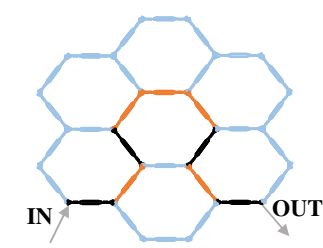

121.5 ps (9 BULs)

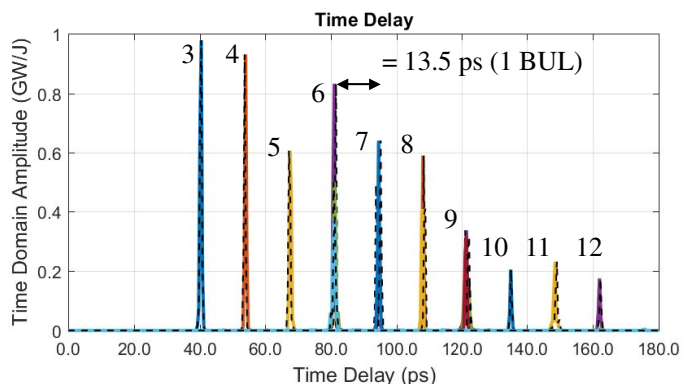

Fig. 3. Discrete optical delay lines. (Top) 7-cell layout and settings for two different time delays. (Bottom) Measured delays up to 12 BULs.

which results in a delay of $67.5 \mathrm{ps}$. When the light-path is modified as in the companion figure, we increase the light-path length to 9 BULs, which corresponds to 121.5 ps. In the right side of the figure, we have included some of the experimental delays obtained by changing the light path length from 3 to 12 TBUs (40.5-162 ps). Our device loss per delay figure is 44.44 $\mathrm{dB} / \mathrm{ns}$. This device would benefit from the integration of an optical amplifier before leaving the chip to compensate the losses in a heterogeneous integration platform.

It could also be possible to employ a larger continuous delay section implemented by overlapping the group delay of two cascaded programmed rings together with the demonstrated discrete delay line, [12].

\section{A. Filtering applications}

In the case of optical filter synthesis, a detailed analysis of mesh topologies and the Basic Unit Length (BUL) implication shows that for hexagonal-mesh-based PICs, the spectral period of the synthetized structures is given by [10]:

$$
\tau=\frac{n_{g} \cdot N \cdot B U L}{c},
$$

where $c$ is the speed of light in vacuum, $n_{g}=4.18$ is the waveguide group index of our Silicon on Insulator (SOI) waveguide, $\mathrm{BUL}=975 \mu \mathrm{m}$, while $N=2,4,6,8, \ldots$ for the synthesis of unbalanced MZIs and $N=6,8,10,12,14,16, \ldots$ for the synthesis of optical ring resonators.

We start by visualizing the time response of two synthesized circuits corresponding to an infinite impulse response filter 6-BUL ORR in Fig. 4, and to a finite impulse response filter 4-BUL MZI in Fig. 5. In the UMZI case depicted in Figure 5.4, we show the temporal response (i.e. a two-tap discrete time filter) corresponding to one of its two outputs. The coupling constant of the TBU acting as the input 
tunable coupler is varied while the one acting at the output coupler is maintained fixed with a coupling ratio of 50:50. In the top/left we can see a case where most of the signal is travelling through the shorter UMZI path.

The inset shows a low extinction ratio in the spectrum response. The remaining figures illustrate how the splitting ratio changes for each contribution when we increase the coupling ratio. When both contributions are equal (like in bottom/left) the highest extinction ratio is achieved. In this case, the coupling ratio is set to compensate the losses in the largest path with respect to the shorter one. The time delay given by the differential path length is 54 ps. The last figure corresponds to the scenario where the contribution from the longer arm in the UMZI is greater than the first one.

In the ORR case, we show the reflection response where the coupling factor is being changed. In the top/left we can see that the TBU performing the tunable coupler is in Bar State. In this case, the signal is not travelling through the ring cavity and is directly transmitted to the output. The inset shows a planar spectrum response since there is not any interferometric structure. The top/right figure shows that if we increase the coupling factor, the different contributions for successive signal recirculation will appear delaying the signal. The overall delay is characterized by a time value given by the propagation across the entire ring cavity length of 6-BULs, $81 \mathrm{ps.} \mathrm{Up} \mathrm{to} 10$ contributions can be appreciated in Fig. 5 top/right. Moving to the bottom/left figure, we can see that we achieve the critical coupling condition resulting in the highest extinction ratio. In this case, we reduce the power of the first contribution and increase the optical power in the ring cavity to overcome the
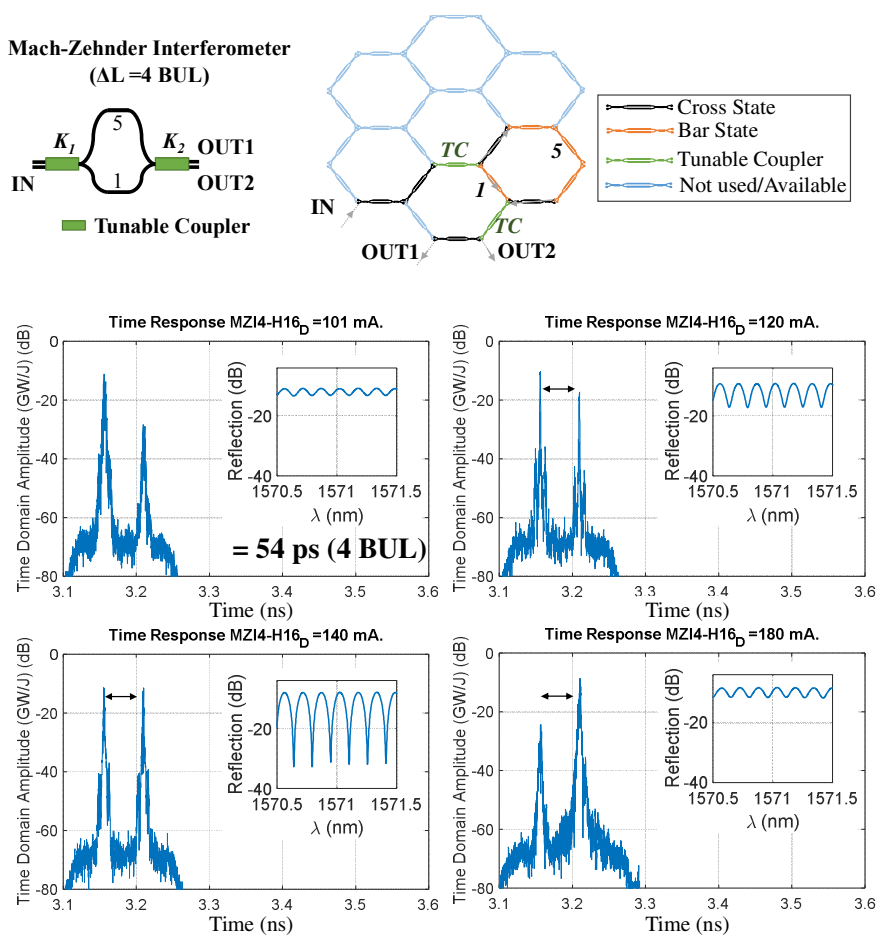

Fig. 4. (Top) Targeted MZI layout to be programmed, 7-cell device setting s (Bottom)Measured time response of an ORR corresponding to 4-BULs. Each figure corresponds to a different input coupling factor. The inset shows the corresponding spectral response.

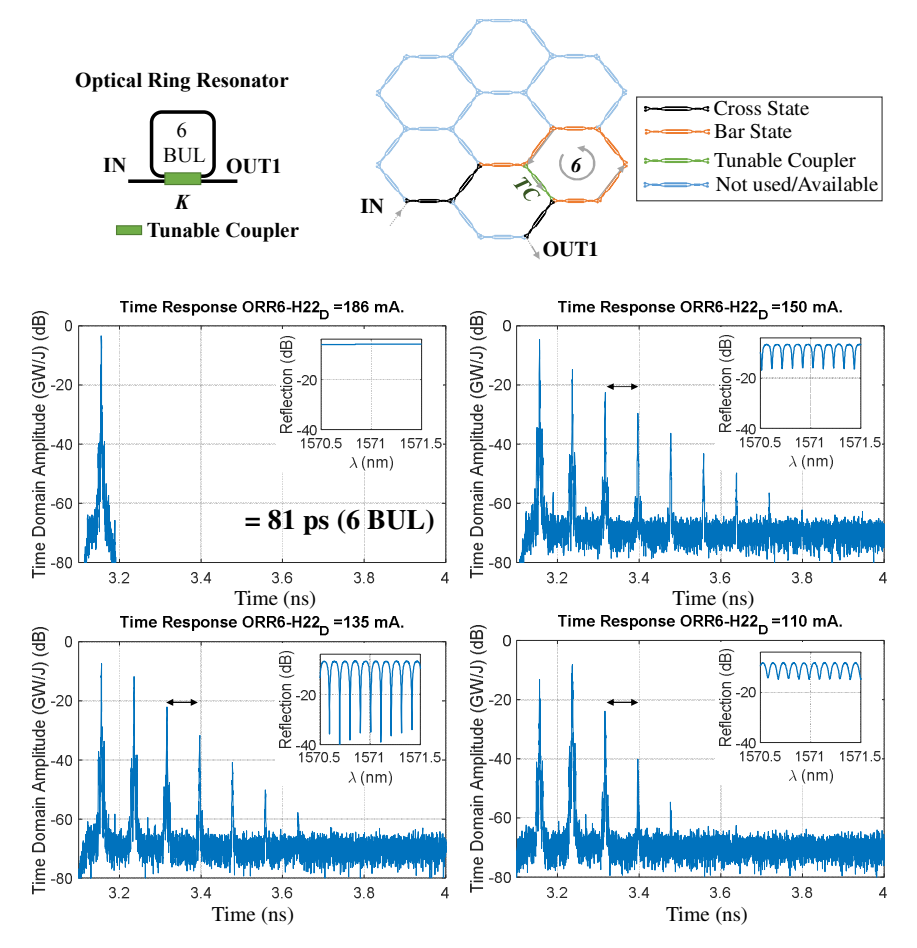

Fig. 5. (Top) Targeted ORR layout to be programmed and 7-cell device setting. (Bottom) Measured time response of an ORR corresponding to 6-BULs. Each figure corresponds to a different coupling factor. The inset shows the corresponding spectral response.

travelling losses inside it. The last figure corresponds to an over-coupled condition. Note that the second contribution is greater than the first one. Both filtering structures show a remarkable extinction ratio greater than $30 \mathrm{~dB}$ if the related couplers are set properly.

\section{B. Beamforming networks based on optical delay lines}

Optical Beamforming applications based on optical delay lines can be as well programmed in our 7-cell core. By configuring some of the TBUs as tunable couplers, it is possible to create adjacent light-paths with an incremental length $\Delta L$. This incremental length will define the tilt angle of the antennas placed at the outputs, together with the distance between the radiating elements. Fig. 6 illustrates two measured cases that implement a different optical delay $\Delta \tau$ corresponding to 2 and 3 BULs.

Fig. 7 illustrates a PIC layout based on a hexagonal waveguide mesh implementing a $1 \mathrm{x} 8$ beamforming network. By suitable configuring the TBUs by the color-code defined in the previous figures, the example illustrates the variation of the differential delay given by path lengths of 4.8 and 10 BULs. Optical Beamforming networks based on providing a differential phase shift to each output can be as well programmed in the hexagonal-mesh core, either by using a tree-scheme with a phase shifter at each output or by programing a linear interferometer for the beamsplitting section. In this case, the output signal frequency and the tilt angle will not be independent. 

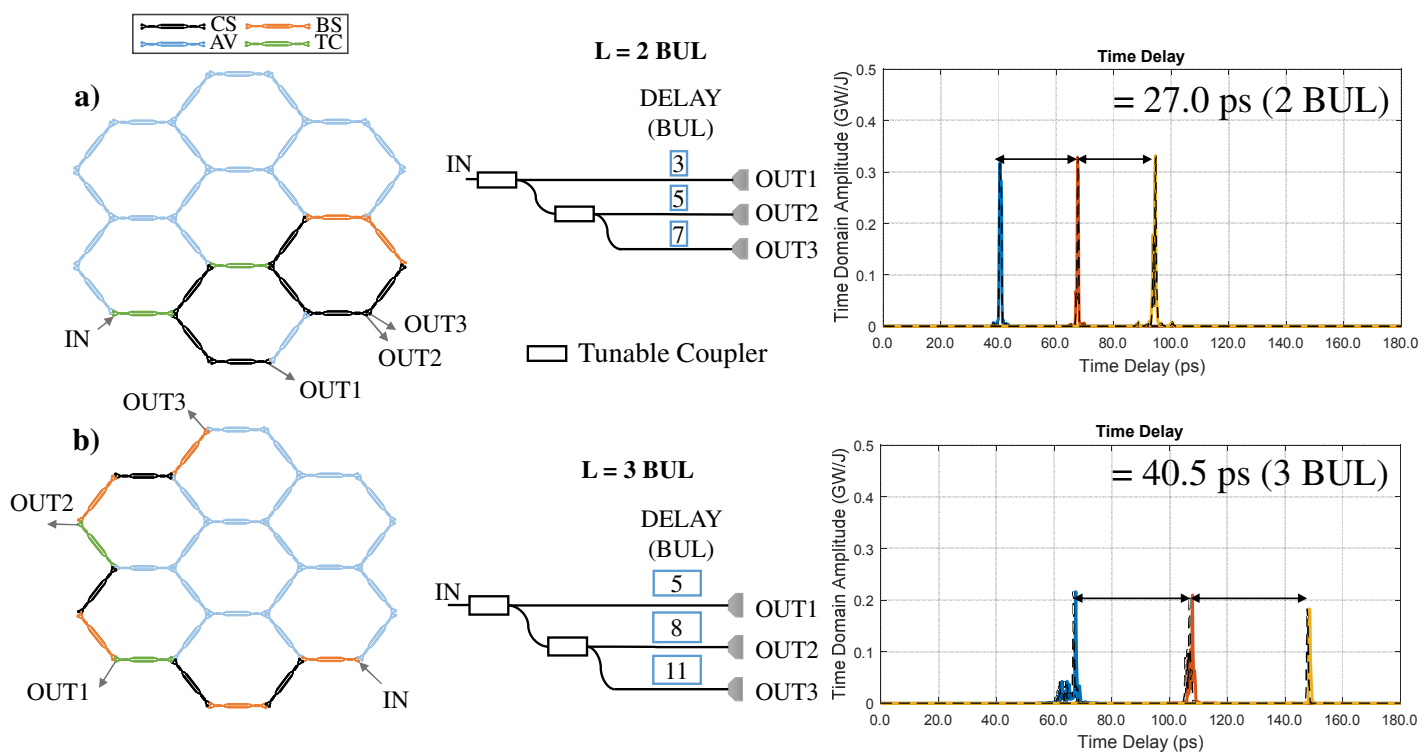

Fig. 6. Measured optical beamformer network: (a) 2-BUL delay, (b) 3-BUL delay: (Left) 7-cell device settings, (Middle) Targeted beamformer layout to be programmed, (right) Measured time delay for each output.
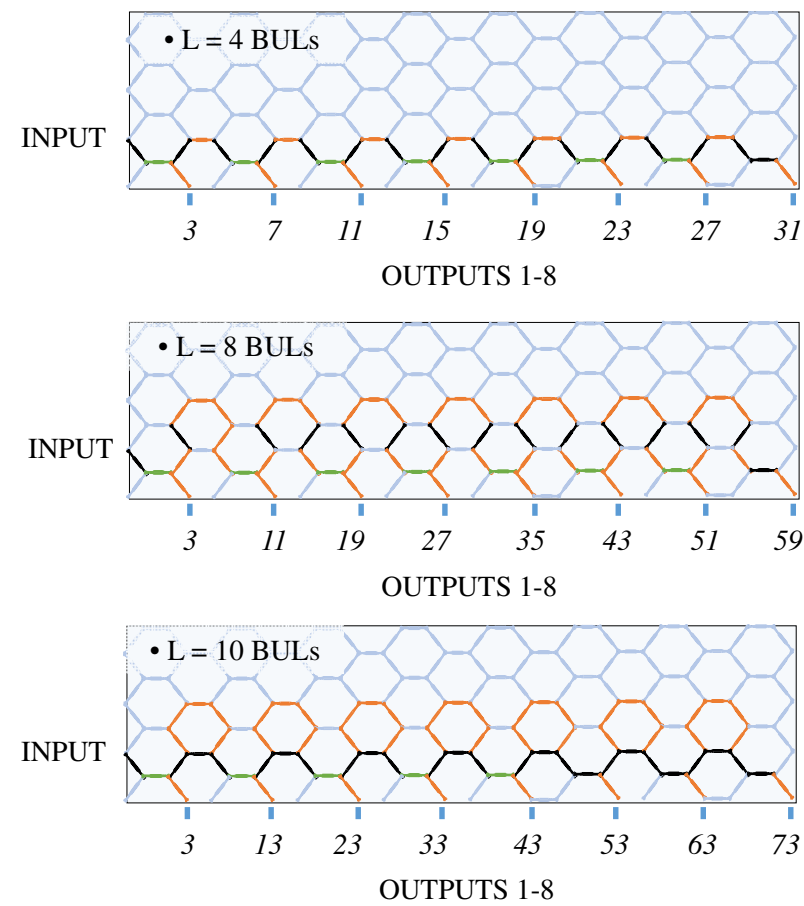

Fig. 7. $1 \mathrm{X} 8$ Beamformer based on discrete optical delay lines: Configuration examples for 4-, 8-, and 10-BUL path difference. Each path length is labbeled at each output.

\section{CONCLUSIONS}

We have reported the implementation of tunable optical time delay lines in an integrated silicon photonics waveguide mesh. We have demonstrated basic circuit topology and circuit parameters reconfiguration enabling reconfigurable optical filters, discrete delay lines and optical beamforming networks.

\section{ACKNOWLEDGMENT}

The authors acknowledge financial support by the ERC ADG-2016 UMWP-Chip, I.G. acknowledges the funding through the Spanish MINECO Ramon y Cajal program. D.P. acknowledges financial support from the UPV through the FPI predoctoral funding scheme. D.J.T.acknowledges funding from the Royal Society for his University Research Fellowship.

\section{R EFERENCES}

[1] J. Capmany and D. Novak, "Microwave photonics combines two worlds," Nat. Photonics vol. 1, pp. 316-330, 2007.

[2] J. Yao, "Microwave photonics," J. Lightwave Technol., vol. 27, no.b3, pp. 314-335,2009.

[3] J. E. Mitchell, "Integrated wireless backhaul over optical access networks," J. Lightwave Technol., vol. 32, no. 20), pp. 3373-3382, 2014.

[4] R. Waterhouse, R. \& D. Novak, "Realizing 5G: Microwave Photonics for 5G Mobile Wireless Systems," IEEE Microw. Mag., vol. 16, pp. 8492, 2015.

[5] D. A. I. Marpaung, C. Roeloffzen, R. Heideman, A. Leinse, S. Sales, and J. Capmany, "Integrated microwave photonics," Laser Photon. Rev., vol. 7, pp. 506-538, 2013.

[6] C. G. H. Roeloffzen, et al, "Silicon nitride microwave photonic circuits," Opt. Express, vol. 21, no. 19, pp. 22937-22961, 2013.

[7] S. Iezekiel, M. Burla, J. Klamkin, D. Marpaung, \& J. Capmany, "RF Engineering Meets Optoelectronics: Progress in Integrated Microwave Photonics,". IEEE Microw. Mag., vol. 16, pp. 28-45, 2015.

[8] D. Pérez, I. Gasulla, \& J. Capmany, "Software-defined reconfigurable microwave photonics processor," Opt. Express, vol. 23, no. 11, pp. 14640-14654, 2015.

[9] L. Zhuang et al., "Programmable photonic signal processor chip for radiofrequency applications," Optica, vol. 2, no. 10, pp. 854-859, 2015.

[10] D. Pérez et al., "Reconfigurable lattice mesh designs for programmable photonic processors," Opt. Express, vol. 24, no. 11, pp. 12093-12106, 2016.

[11] C. K. Madsen \& J. H. Zhao, Optical Filter Design and Analysis: A Signal Processing Approach, Wiley (1999).

[12] X. Wang et al., "Continuously tunable ultra-thin silicon waveguide optical delay line," Optica, Vol. 4, no. 5, p. 507, 2017. 